\title{
Development of surface relief on polycrystalline metals due to sputtering
}

\author{
V.S. Voitsenya ${ }^{1}$, M. Balden ${ }^{2}$, A.F. Bardamid ${ }^{3}$, V.N. Bondarenko ${ }^{1}$, J.W. Davis ${ }^{4}$, V.G. Konovalov ${ }^{1}$, \\ I.V. Ryzhkov' ${ }^{1}$, O.O. Skoryk ${ }^{1}$, S.I. Solodovchenko ${ }^{1}$, Zhou Zhang-jian ${ }^{5}$ \\ ${ }^{1}$ IPP NSC KIPT, 61108 Kharkov, Ukraine; \\ ${ }^{2}$ Max-Planck-Institut für Plasmaphysik, EURATOM Association, Garching, Germany \\ ${ }^{3}$ Taras Shevchenko National University, 01033 Kiev, Ukraine; \\ ${ }^{4}$ University of Toronto Institute for Aerospace Studies, 4925 Dufferin St., Toronto, ON, Canada M3H5T6; \\ ${ }^{5}$ University of Science and Technology Beijing, Beijing 100 083, China
}

\begin{abstract}
The characteristics of surface microrelief that appear in sputtering experiments with polycrystalline metals of various grain sizes have been studied. Specimens with grain sizes varying from $30-70 \mathrm{~nm}$ in the case of crystallized amorphous alloys, to 1 $-3 \mu \mathrm{m}$ for technical tungsten grade and $10-100 \mu \mathrm{m}$ for recrystallized tungsten were investigated. A model is proposed for the development of roughness on polycrystalline metals which is based on the dependence of sputtering rate on crystal orientation. The results of the modeling are in good agreement with experiments showing that the length scale of roughness is much larger than the grain size.
\end{abstract}




\section{Introduction}

Under the influence of high-fluence ion bombardment highly-polished mirrors fabricated from polycrystalline metals are observed to develop a complex step structure relief [1-3], attributed to the difference of the sputtering yield of grains with different crystal orientation relative to the surface [3]. The relief can be characterized with modern microscopy methods as optical interference microscopy, confocal laser scanning microscopy (CLSM), atomic force microscopy (AFM), scanning tunneling microscopy (STM), and scanning electron microscopy (SEM) with electron backscatter diffraction (EBSD). The longitudinal size of the developed roughness, i.e., the scale length of the step features, is determined by the grain size, and it would be expected that the scale length for the roughness would be similar to the grain size, as suggested in [4]. However, in experiments with $\mathrm{Rh}$ film mirrors [5] it was found that the sputtering of very fine-grain mirrors led to surface roughness with a longitudinal scale noticeably exceeding the grain size.

As a consequence, the degradation of optical reflectance due to the development of largescale $(1-10 \mu \mathrm{m})$ surface relief can occur for fine grain mirrors, even though the mirror grain size is much less than the wavelength of the light ( $\sim 0.1 \mu \mathrm{m}$ in the case of $\mathrm{Rh}$ film mirrors).

A similar lack of direct correlation between the size of grains and characteristics of surface roughness was found in recent experiments with specimens made of a nano-scale metal alloy, fine-grain $\mathrm{Cu}$ alloy, fine-grain $\mathrm{Mo}$ and $\mathrm{W}$, technical small-grain $\mathrm{W}$ and recrystallized $\mathrm{W}$ specimens.

In the present paper, we compile and analyze the results of some of our experiments on this subject (in [5-7] and some new data) and make comparisons with numerical simulations of surface roughness development on polycrystalline materials with single- or multi-sized grains having randomly distributed crystal orientation.

\section{Experimental technique}

The experiments were carried out with specimens having different sizes of crystallites: crystallized amorphous $\mathrm{Zr}_{41.2} \mathrm{Ti}_{13.5} \mathrm{Cu}_{12.5} \mathrm{Ni}_{10} \mathrm{Be}_{22.5}$ alloy (crystallite particle size $30-70 \mathrm{~nm}$ ), $\mathrm{Rh}$ film on $\mathrm{Cu}$ substrate mirrors (size of crystallites $\sim 100 \mathrm{~nm}$ ), fine-dispersed alloy $\mathrm{Cu}-\mathrm{Cr}-\mathrm{Zr}$ (size 200-300 nm), fine-grain molybdenum and tungsten (250-350 nm), "ITER-grade" tungsten (1$3 \mu \mathrm{m})$, and recrystallized tungsten $(\geq 10 \mu \mathrm{m})$. All specimens were prepared as mirrors of high optical quality; detailed descriptions are given below.

Sputtering was performed by exposing specimens to plasma ions in a double mirror magnetic configuration [1]. Electron cyclotron resonance plasmas with densities of $\sim 10^{16}$ ions $/ \mathrm{m}^{3}$ and electron temperature of $\sim 5 \mathrm{eV}$ in deuterium or argon were used as a source of incident particles. Ions were accelerated normal to the specimen surface by biasing the specimen holder with a negative potential. Due to the low sputtering yield for deuterium ions on W, Ar plasmas were used for $\mathrm{W}$ specimens to reduce the required sputtering time. Prior to any optical measurements, specimens were cleaned by a short exposure, $\sim 2 \cdot 10^{23}$ ion $/ \mathrm{m}^{2}$, to low energy, $\sim 60$ 
$\mathrm{eV}$, deuterium ions. (In some cases, an even shorter exposure to low energy Ar plasma ions was applied for cleaning instead.)

The reflectance at normal incidence in the wavelength range $220-650 \mathrm{~nm}$, and the mass loss due to sputtering (to give the average sputter depth $\Delta \mathrm{h}$ ) was measured after exposures (more details in [1]). Thus the rate of degradation of optical properties on the thickness of sputtered layer was found. Surface characteristics were further analyzed by the use of optical microscopies, SEM, AFM and STM.

\section{Details of simulation}

The processing of AFM and STM data was performed by means of our own software created in Mathcad, based on the principles given in the manual of the analytic software "FemtoScan Online", version 2.3.134 [8]. The following parameters characterizing surface roughness after sputtering procedures were used:

- $\mathrm{R}_{\mathrm{q}}$ is the root mean square deviation of the heights in a discrete microrelief profile from the trend-line to that profile.

- $\mathrm{S}_{\mathrm{m}}$ is the mean period of longitudinal wave in a microrelief profile. Following the definition in [8], the local period $S_{i}$ is the distance between two points where the profile intersects (from below upwards) the trend-line to the profile. $\mathrm{S}_{\mathrm{m}}$ is an arithmetic average of all local periods $\mathrm{S}_{\mathrm{i}}$, thus characterizing the whole profile.

The detailed explanation of $\mathrm{R}_{\mathrm{q}}, \mathrm{S}_{\mathrm{m}}$, longitudinal wavelengths distribution $\Delta \mathrm{N} / \Delta \lambda$ (number of longitudinal periods $\Delta \mathrm{N}$ per wavelength interval $\Delta \lambda$ ), the interval of longitudinal wavelengths $\lambda_{\mathrm{I}}$, and the most probable wavelength $\lambda_{0}$ in distribution is given in the Appendix. The spiral track reading approach and the problem of short-wave noise effects in the profiles are also discussed there.

The parameters $\mathrm{R}_{\mathrm{q}}$ and $\mathrm{S}_{\mathrm{m}}$ are always present in traditional analysis of surface roughness. In particular, $\mathrm{S}_{\mathrm{m}}$ is similar in nature to the autocorrelation length that traditionally characterizes the linear dimension of irregularities of rough surface, see e.g., $\S 6$ in [9]. The formulas of reflectance coefficients for mirrors with rough surfaces requires such data $[9,10]$, but for us $S_{m}$ itself is of primary importance.

In our earlier work [5] devoted to mirrors fabricated as $\mathrm{Rh}$ films on $\mathrm{Cu}$ substrate (see chapter 4.2 below in the text) we found longitudinal wavelength spectra using the Fourier spectrum of height profile $\mathrm{h}(\mathrm{x})$ of three specimens. Recently we proposed that the longitudinal wavelength $\Lambda$ corresponding to harmonic with the highest amplitude in a Fourier spectrum could be adopted as the scale length characterizing the longitudinal wavelength. But this parameter was not found to have a direct correlation to $S_{m}$ and thus, the longitudinal wavelength, $\Lambda$, has not been used in our current analysis. Instead, we have used only an analysis of wavelength distributions $\Delta N / \Delta \lambda$ and of the related parameters. 


\section{Results}

\subsection{Crystallized amorphous alloy mirrors}

A comparison of sputtering behavior and optical characteristics of amorphous and crystallized mirror specimens fabricated by annealing from an amorphous alloy with composition $\operatorname{Zr}(41.2 \%) \mathrm{Ti}(13.8 \%) \mathrm{Cu}(12.5 \%) \mathrm{Ni}(10 \%) \mathrm{Be}(22.5 \%)$, in atomic percents, was presented in [6]. After crystallization by annealing at $773 \mathrm{~K}$ for $1 \mathrm{~h}$, the position of the peaks in a diffractogram indicated the existence of the following nanocrystals: $\mathrm{Zr}_{2} \mathrm{Ni}, \mathrm{Ti}_{2} \mathrm{Ni}, \mathrm{Zr}_{2} \mathrm{Cu}$, and $\mathrm{Zr}_{x} \mathrm{Cu}_{y}$ with $x$ and $y$ exceeding two. For the amorphous mirrors, $\mathrm{Ar}^{+}$sputtering to an averaged sputter depth of $13.4 \mu \mathrm{m}$ was found to have little impact on the optical characteristics [6]. However, the optical properties of the crystallized mirrors were found to degrade very rapidly by sputtering. Following the sputtering of the $8.9 \mu \mathrm{m}$ thick layer, the crystallized specimen became rather rough, with the roughness being non-uniform along the surface. The SEM photo of this specimen in Fig. 1 shows the region of the surface with the highest roughness (by visual inspection). The scale of the longitudinal wavelength of inhomogeneity is on the order of $1 \mu \mathrm{m}$ (as can be seen by eye from this figure).

In order to make a comparison between the surface roughness scale length and the size of the crystals in the original specimen, the crystallites sizes must be determined. This can be done by evaluating the diffraction peaks in the X-ray diffractogram (see Fig. 1 in [11]) and using the formula [12]:

$$
D=\frac{0.9 \lambda}{\beta \cos \theta}
$$

where $\lambda$ is the X-ray wavelength, $\theta$ is the Bragg angle, and $\beta$ is the FWHM of the diffraction peak.

The results of this evaluation are shown in Table 1 giving a maximum crystallite size of $\sim 70$ $\mathrm{nm}$. This is an order of magnitude smaller than the observed scale of longitudinal wavelength of surface roughness, $\sim 1 \mu \mathrm{m}$. Such a large difference is difficult to explain from existing sputtering models, e.g. $[13,14]$.

Note, similar inconsistencies are observed between the grain sizes in polycrystalline metals and the mean longitudinal period $\mathrm{S}_{\mathrm{m}}$ observed following sputtering as discussed below. All data are summarized in Table 2.

\subsection{Rh film on $\mathrm{Cu}$ substrate mirror specimens}

The surface analysis of three $\mathrm{Rh}$ film mirror specimens on $\mathrm{Cu}$ substrates was first presented in [5], but the results have been re-analyzed following the approach of the present paper. The specimens had different resistances to sputtering by deuterium plasma ions with a wide energy distribution. Because of this, they were sputtered to different depths: a) $0.5, b$ ) 1.1 , and c) $2.3 \mu \mathrm{m}$. The surface roughness of the specimens is noticeably reduced from (a) to (c).

Due to the small size of the region analyzed by STM, $1.6 \times 1.6 \mu \mathrm{m}^{2}$ or $1.1 \times 1.1 \mu \mathrm{m}^{2}$ for these specimens, better statistics were obtained by the spiral track approach, which allowed track lengths to be significantly larger than the dimensions of the analysis area. Based on three 
microphotographs of every specimen and on the calculated distributions $\Delta \mathrm{N} / \Delta \lambda$, we obtained the longitudinal parameters, in $\mu \mathrm{m}$ : a) $\mathrm{S}_{\mathrm{m}}=0.27-0.3, \lambda_{\mathrm{I}}=0.02-1.0, \lambda_{0}=0.1-0.25$; b) $\mathrm{S}_{\mathrm{m}}=0.2, \lambda_{\mathrm{I}}=0.02-$ $0.7, \lambda_{0}=0.18$; c) $\mathrm{S}_{\mathrm{m}}=0.15-0.2, \lambda_{\mathrm{I}}=0.016-1.0, \lambda_{0}=0.07-0.11$. The values of $\mathrm{S}_{\mathrm{m}}$ exceed the typical longitudinal dimensions of the Rh crystallites $\sim 0.1 \mu \mathrm{m}$ for all 3 specimens (Table 2).

\subsection{Copper alloy mirrors}

Copper alloys with chromium and zirconium were transformed into fine grain materials by intensive plastic deformation [15]. This led to the production of billets with a highly-faulted structure across the whole billet with grain sizes of $200-300 \mathrm{~nm}$. From this material, mirrors in the shape of $20 \mathrm{~mm}$ diameter disks of $5 \mathrm{~mm}$ thickness were prepared for optical measurements.

Prior to the optical measurements, specimens were cleaned by exposing to ions from a deuterium plasma. After the initial optical characterization, specimens were exposed to a series of 10 min exposures to deuterium plasma ions with a wide energy distribution $(100-1350 \mathrm{eV})$, achieved by varying the bias on the specimen holder [1]. The current density was $2.8 \mathrm{~mA} / \mathrm{cm}^{2}$, the thickness of the sputtered layer reached $\Delta \mathrm{h} \sim 4.6 \mu \mathrm{m}$. Details of results can be found in [7].

Subsequent analysis of the surface microrelief was performed with AFM on a $50 \times 50 \mu \mathrm{m}^{2}$ area. This gave $S_{\mathrm{m}}=6 \mu \mathrm{m}$ for the longitudinal inhomogeneity of the microrelief due to sputtering. In the distribution $\Delta \mathrm{N} / \Delta \lambda$ the wavelengths were present in the range $\lambda_{\mathrm{I}}=1-26 \mu \mathrm{m}$, the most probable value $\lambda_{0}=3.6 \mu \mathrm{m}$. Again as for specimens noted above, this is more than one order of magnitude larger than the grain size.

\subsection{Mo and $W$ mirror specimens}

Fine grain Mo and $\mathrm{W}$ materials were fabricated using a resistance sintering procedure [16], resulting in grain sizes of $250-350 \mathrm{~nm}$ (e.g., one of four unpolished W specimens is shown in Fig. 2). During the sintering process, large currents are passed through specimens in a very short time, leading the melting and bonding of some of the grains. The average size of such conglomerates is approximately $1-2 \mu \mathrm{m}$; thus, SEM images do not always reveal clear grain boundaries. After polishing and the usual D plasma cleaning procedure, the spectral reflectance at normal incidence in the range $220-650 \mathrm{~nm}$ was found to agree closely with the nominal values for Mo and W [17]. However, the reflectance was strongly degraded by sputtering with deuterium ions of a broad energy distribution $(100-1350 \mathrm{eV})$ for Mo specimens, or $600 \mathrm{eV} \mathrm{Ar}^{+}$ ions for $\mathrm{W}$ specimens, see Fig. 3.

The reduction in reflectance implies the development of surface roughness, and this was analyzed by AFM over an area of $50 \times 50 \mu \mathrm{m}^{2}$. For two Mo mirrors the mean longitudinal period was $\mathrm{S}_{\mathrm{m}}=4.8 \mu \mathrm{m}$, following the sputter removal of $\sim 1.7 \mu \mathrm{m}$. The range of longitudinal wavelengths was $\lambda_{\mathrm{I}}=0.3-25 \mu \mathrm{m}$ in the distribution $\Delta \mathrm{N} / \Delta \lambda$ for both specimens. The most probable wavelength $\lambda_{0}=3.5 \mu \mathrm{m}$.

The corresponding average values for four $\mathrm{W}$ specimens were: $\mathrm{S}_{\mathrm{m}}=3 \mu \mathrm{m}$, and $\lambda_{\mathrm{I}}=0.2-17$ $\mu \mathrm{m}, \lambda_{0}=1.5 \mu \mathrm{m}$, following the removal of $\sim 1 \mu \mathrm{m}$. For both materials, the scale characterizing the longitudinal wavelength of the roughness is, again, much larger than the grain size. 


\subsection{Mirrors from Japanese "ITER-grade" tungsten}

A description of the A.L.M.T. Corp. (Japan) "ITER-grade" tungsten is given in [18]. It is a small-grain tungsten, with grains preferentially oriented with their largest dimension $(\sim 5 \mu \mathrm{m})$ perpendicular to the surface to improve thermal transport. Parallel to the surface, the grain size is $1-3 \mu \mathrm{m}$ (see Fig. 1 in [18]).

As is shown in Fig. 4, the surface relief after sputtering more closely resembles alternating mountain ridges and valleys with typical distances between them of $7-20 \mu \mathrm{m}$. Different regions of this macroscopic structure vary in micro-scale roughness. In the topmost of the AFM photograph, inside a region of the wide "valley", one can obtain $\mathrm{S}_{\mathrm{m}}=5.3 \mu \mathrm{m}, \lambda_{0}=3 \mu \mathrm{m}$ and $\lambda_{\mathrm{I}}=$ $1-12 \mu \mathrm{m}$. Thus, the local microrelief in a wide "valley" (also in "ridges" as can be seen in Fig. 4) is generally characterized by longitudinal inhomogeneities that appear to correlate more-orless to the grain sizes. Nevertheless, the overall surface roughness is dominated by the larger scale "gross-microrelief".

\subsection{Recrystallized tungsten}

Tungsten specimens with maximal grain sizes far larger than $10 \mu \mathrm{m}$ were prepared by initially fabricating double-sided mirror specimens from polycrystalline $\mathrm{W}$, then allowing them to recrystallize at $2073 \mathrm{~K}$ for $1 \mathrm{~h}$ [19]. A $\sim 3.9 \mu \mathrm{m}$ thick layer was then sputtered from such specimens with $600 \mathrm{eV} \mathrm{Ar}^{+}$ions. The EBSD data for a $1 \times 1 \mathrm{~mm}^{2}$ region of the surface is shown in Fig. 5, indicating the orientation of the crystallites. There is a clear tendency of similar orientations to form groups of varying sizes, thus contributing to long wavelength surface roughness. The height steps observed between crystallites with similar orientations $(20-100$ $\mathrm{nm}$ ) are much smaller than between grains with different orientations (maximum $\Delta \mathrm{h} \approx 1.5 \mu \mathrm{m}$ ).

Processing the 3D information of CLSM for this specimen clearly demonstrates the appearance of a "long-wave" inhomogeneity of the relief exceeding the size of the grains. In this respect the results for this "conventional" polycrystalline specimen are qualitatively very similar to the results obtained for the fine-grain specimens. The analysis of distributions $\Delta \mathrm{N} / \Delta \lambda$ at the smoothing parameter $m=9$ (see Fig. 6) resulted in the parameters $S_{m}=150 \mu \mathrm{m}, \lambda_{\mathrm{I}}=2-1150 \mu \mathrm{m}$, and $\lambda_{0}=95 \mu \mathrm{m}$. One can see that $\mathrm{S}_{\mathrm{m}}$ exceeds the size of grains $(10-100 \mu \mathrm{m})$, and the longest wavelengths of inhomogeneity is an order of magnitude longer than the size of largest grains.

For this polycrystalline W specimen, the (110) grains were found to have the lowest resistance to sputtering, while the (111) grains had the highest resistance [20]. This appears to be contradictory to results for stainless steel in [3], where the (111)-oriented grains were found to have the highest sputtering yield. However, these metals belong to different crystal structure, namely $\mathrm{W}$ is a bcc (body-centered cubic) metal, while SS is a fcc (face centered cubic) metal. Thus the densest faces are (110) and (111) for W and SS, respectively. 


\section{Results of modeling of surface relief development}

We begin by assuming that the main reason for the appearance of microrelief during the sputtering of polycrystalline materials is the difference in the sputtering rate between crystals which have different orientations of their crystallographic axes relative to the surface normal [21]. Such differences have been observed experimentally for copper [1] and stainless steel [2,3] mirrors subjected to sputtering by deuterium ions, and for $\mathrm{W}$ mirror sputtered with Ar ions [20]. It was also found that the differences in sputtering rates increased with increasing ion energy [1, 2].

In a given material, the distribution of grain orientations is likely to depend on the fabrication process. This distribution may be random, or there could be regions where particular orientations are favored, if some specific measures are used to form a textured material. Even in the case of a random directional distribution $\left(0-360^{\circ}\right)$, there is a probability that some number of grains with similar orientations will form a local group with approximately the same sputtering yield. Some distance away, another group might appear with orientations clustered around another crystallographic axis. The mean distance between such groups might then be a factor in determining the scale that characterizes the longitudinal wavelength of inhomogeneity.

We begin our model by assuming, for simplicity, that all crystallites have identical surface area and shape (square, $1 \times 1 \mu^{2}$, this constraint will be relaxed somewhat later) and initial height, but their crystallographic axes are randomly distributed. We assume that if the inclination of grain orientation from a given low-indexed crystallographic axis is small, the sputtering rate will be similar to grains oriented exactly along the given axis. How far a crystallite can vary from the nominal orientation without a significant change to the sputtering yield is not known, but for these calculations, we have assumed a range of $\pm 15^{\circ}$. Thus, all grains which have an inclination from the (111) face of $<15^{\circ}$ are assumed to have the same sputtering rate as the (111) face oriented exactly along the surface. Also a rotation of $180^{\circ}$ will give the same sputtering rate due to the lattice symmetry.

Thus, our model effectively involves 6 different orientations, including the 3 primary directions of cubic crystal structure, (111), (100) and (110), along with three intermediate ones. The actual sputtering yields for each orientation are not known, so we assign yields to all faces of $\mathrm{Y}=0.1,0.2,0.3,0.4,0.5,0.6$. Smaller variations in sputtering yield would mean that a greater sputter depth would be required to achieve the same level of surface roughness, but this would not change the overall result. Thus, the height of each grain decreases in proportion to Y, e.g., by $1 \mathrm{~nm}$ for $\mathrm{Y}=0.1$, etc. An example of a "post-sputtering surface" with identically-sized square grains of 1 length unit size in $45 \times 40$ grid is shown in Fig. 7 . Note the length unit in the modeling is arbitrary and is set to $\mu \mathrm{m}$ in the following for demonstrative description. Calculations typically included up to 8100 crystallites on a $90 \times 90 \mu \mathrm{m}^{2}$ grid.

Initially, the "mirror" surface is ideally smooth, with all grains having an identical height. The sputtering process removes $\Gamma_{0} \times \mathrm{Ynm}\left(\Gamma_{0}\right.$ is the fluence of projectiles, uniform along the surface of "mirror"), thus the face with $\mathrm{Y}=0.6$ is sputtered the greatest, while the face with $\mathrm{Y}=$ 0.1 will be sputtered to $1 / 6$ of that depth. 
To find the relief characteristics, height "measurements" were sampled along a rectangular spiral, starting from the centre of the grid ("mirror") and extending to the grid boundary. The total length of the path was $\sim 1350 \mu \mathrm{m}$. The data were processed to determine the rms roughness, $\mathrm{R}_{\mathrm{q}}$ and the distributions of longitudinal wavelengths of the surface relief.

In addition to the case with identically-sized square crystallites in the $90 \times 90 \mu^{2}$ grid, 3 other "materials" were analyzed to determine the role of varying grain size on the development of surface relief, i.e., to model more closely real polycrystalline materials:

i. only $1 \mu \mathrm{m} \times 1 \mu \mathrm{m}$ grains, with random orientation distribution.

ii. $2 \mu \mathrm{m} \times 2 \mu \mathrm{m}$ grains randomly cover half of the $90 \times 90 \mu \mathrm{m}^{2}$ matrix; the remaining area is randomly covered with $1 \mu \mathrm{m} \times 1 \mu \mathrm{m}$ grains.

iii. $3 \mu \mathrm{m} \times 3 \mu \mathrm{m}$ grains and $1 \mu \mathrm{m} \times 1 \mu \mathrm{m}$ grains; each type covers half of the area.

iv. $4 \mu \mathrm{m} \times 4 \mu \mathrm{m}$ grains and $1 \mu \mathrm{m} \times 1 \mu \mathrm{m}$ grains; again, each type covers half of the area.

Fig. 8 illustrates the case of color map of the last combination. To improve statistics for the parameter determination, 6 mirror surfaces were generated and analyzed for each of the 4 grainsize combinations. For each of the four cases ( $i-i v)$, the longitudinal periods distributions $\Delta \mathrm{N} / \Delta \lambda$ were averaged over the 6 generated specimen surfaces and the results are presented in Fig. 9. In addition to surface variations comparable to the $1 \times 1 \mu \mathrm{m}^{2}$ grain size, the longitudinal components of the surface roughness with a much larger scale length are clearly seen.

For all 4 cases, the $\mathrm{R}_{\mathrm{q}}$ value is $\sim 1.7 \mathrm{~nm}$ assuming that $\Gamma_{0}$ leads to sputtering depths 1 to $6 \mathrm{~nm}$ for every face with $Y=0.1$ to 0.6 , respectively. The near identical values of $R_{q}$ in the 4 cases are due to the nearby equal mean yield. It is clearly seen from Fig. 8 that there are groups of grains with similar orientations. In this sense, this 2-grain-size example qualitatively resembles Fig. 5 with grain sizes varying over a wide range.

The distribution of the longitudinal periods, $\Delta \mathrm{N} / \Delta \lambda$, is shown in Fig. 9. As these artificial profiles, $\mathrm{h}(\mathrm{x})$, contain no "measurement noise", there was no need to smooth the profiles. The analysis of distributions resulted in the minimal $\lambda_{\mathrm{I}, \min }=2 \mu \mathrm{m}$ in all cases due to the minimal size of the " $\Delta \lambda$ bin". Here $\lambda_{0}$ coincides with $\lambda_{\mathrm{I}, \mathrm{min}}$ in cases $\mathrm{i}$, iv, and is slightly larger in cases ii, iii.

\section{Summary}

The experimental results and the results of modeling support the hypothesis that differences in sputtering rates for grains with different crystal orientations leads to development of surface roughness with longitudinal scale-lengths much larger than the individual grain sizes. Experimentally, this result is independent of the grain size over the range from tens of $\mathrm{nm}$ (crystallized amorphous mirror specimen) to tens of $\mu \mathrm{m}$ (polycrystal $\mathrm{W}$ mirror). It is true, even for the case of identically-sized crystallites distributed with random orientations. (In calculations, we used a nominal grain size of $1 \mu \mathrm{m} \times 1 \mu \mathrm{m}$, however, the results could be scaled to crystallites of any size.)

The development of the relief with "valleys" and "ridges" on the surface of "ITER-grade" specimens is probably related to the complex processing of this material. Because of large 
differences in the sputtering yields of these characteristic features of microrelief $(\Delta \mathrm{h} \sim 1.5 \mu \mathrm{m}$ as one can see in Fig.4), and taking into account the results of EBSD measurements for recrystallized tungsten, the results suggest that most of grains in the "valleys" have orientation close to (111), and the grains in the "ridges" are mainly orientated along the (110) axis. Therefore the "interior" microrelief, within the "valleys" or "ridges", is not much different from the grain size, like in the groups of grains with close orientation in the case of recrystallized W specimens.

Even in the absence of mechanisms (either deliberate or naturally-occurring) to locally order grains with some particular orientation (i.e. for any non-ordered grain orientation, either chaotic or isotropic), there is a high probability that grains with small differences in orientation (and therefore similar sputtering rate) will be found in local groupings (Fig. 7). The consequence of such grouping of grain orientations is the development, due to sputtering, of larger-scale roughness much longer than the size of an individual grain. Properties such as porosity and grain boundaries, as well as non-normal incidence of sputtering particles, may also play some role, which was beyond the scope of this paper.

The results of this study have application in the choice of material and structure for in-vessel mirrors for optical plasma diagnostics in ITER. Here, the requirement for mirrors with line-ofsight views of the plasma is that the characteristic scale length of surface roughness developing due to sputtering by charge exchange atoms should be significantly less than the wavelength of the light being analyzed. It had been proposed [4] that the use of thin film mirrors or nanocrystalline mirrors with grain sizes much smaller than the required wavelength may be suitable for such applications. For example, for visible or near-infrared ranges of the spectrum mirrors with crystallite sizes $\leq 70 \mathrm{~nm}$ looked like could be acceptable. However, both modeling and experiments suggest that this approach will not be effective. Single crystal mirrors (e.g., [23]) remain the best possibility for these applications. 


\section{References}

1. A.F. Bardamid, V.T. Gritsyna, V.G. Konovalov, et al. Ion energy distribution effect on degradation of optical properties of ion bombarded copper mirrors. Surf. Coatings Technol., 103-104 (1998) 365.

2. A. Bardamid, V. Bryk, V. Konovalov et al. Erosion of steel under bombardment with ions of a deuterium plasma. Vacuum, 58 (2000) 10-15.

3. M. Balden, A.F. Bardamid, A.I. Belyaeva, K.A. Slatin, J.W. Davis, A.A. Haasz, M. Poon, V.G. Konovalov, I.V. Ryzhkov, A.N. Shapoval, V.S. Voitsenya. Surface roughening and grain orientation dependence of the erosion of polycrystalline stainless steel by hydrogen irradiation. J. Nucl. Mater., 329-333 (2004) 1515-1519.

4. V.S,Voitsenya, A.F.Bardamid, M.F.Becker V.T.Gritsyna et al. Some problems of the material choice for the first mirrors of plasma diagnostics in a fusion reactor. Rev. Sci. Instr., 70 (1999) 790-793.

5. V.N. Bondarenko, A.F. Bardamid, V.S. Voitsenya, V.G. Konovalov, S.P. Kulyk, S.I. Solodovchenko, K.I. Yakimov, M.V. Dobrotvorskaya. Effects of long-term ion bombardment on some optical properties of Rh film mirrors and bulk polycrystalline mirrors. Problems of Atomic Science and Technology, series: Plasma Physics, 2006, \#6, pp. 80-83. http://vant.kipt.kharkov.ua/tabframe.html

6. V.S. Voitsenya, A.F. Bardamid, A.I. Belyaeva, V.N. Bondarenko, A.A. Galuza, V.G. Konovalov, I.V. Ryzhkov, A.A. Savchenko, A.N. Shapoval, A.F. Shtan', S.I. Solodovchenko, K.I. Yakimov. Modification of optical characteristics of metallic amorphous mirrors under ion bombardment. Plasma Devices and Operations. Vol. 17, No. 2, June 2009, 144-154.

7. A.I. Belyaeva, I.V. Kolenov, A.A. Savchenko et al. Influence of grain size on resistance to ion sputtering of mirrors from low chromium-zirconium copper alloy. Problems of Atomic Science and Technology, series Nuclear Fusion, 2011, №4, pp. 50-59. http://vant.iterru.ru/vant 2011 4/5.pdf (In Russian).

8. Scanning Probe Microscopy Image Processing Software User's Manual "FemtoScan Online". Version 2.3.134 - A.S. Filonov, A.D. Sushko I.V. Yaminsky - Moscow: Advanced Technologies Center, 2011, 154 pp. (In Russian).

9. I. Ohlídal, D. Franta, Ellipsometry of thin film systems, in: E. Wolf (Ed.), Progress in Optics, vol. 41, North-Holland, Amsterdam, 2000, pp. 181-282.

10. D. Franta, I. Ohlídal, Comparison of effective medium approximation and Rayleigh-Rice theory concerning ellipsometric characterization of rough surfaces / Optics Communications 248 (2005) 459-467.

11. A.F. Bardamid, V.S. Voitsenya, J.W. Davis, V.G. Konovalov, K.V. Kovtun, I.V. Ryzhkov, A.F. Shtan', S.I. Solodovchenko, O.V. Trembach, A.A. Vasil'ev, U.Breuer, A.Litnovsky. Comparison of the behavior of $\operatorname{Zr}(41.2 \%) \mathrm{Ti}(13.8 \%) \mathrm{Cu}(12.5 \%) \mathrm{Ni}(10 \%) \mathrm{Be}(22.5 \%)$ amorphous and crystallized mirrors under deuterium ion bombardment. Journal of Alloys and Compounds, 514 (2012) 189-194.

12. G.W. Brindley, G. Brown, Crystal Structures of Clay Minerals and their X-ray Identification. Mineralogical Society, London, 1980, p. 131.

13. I.H. Wilson, J. Belson, O. Auciello. Secondary effects in ion bombardment - induced surface erosion. In the book: Ion Bombardment Modification of Surfaces. Fundamentals and Applications. Edited by O. Auciello, R Kelly. (Elsevier 1984), Chapter 6.

14. Z.W. Kowalski. Ion-bombardment modification of the surface morphology of solids. Part 1. Change of surface roughness. J. Mater. Sci., 35 (1990) 3675-3679.

15. R.Z. Valiev, I.V. Aleksandrov. Nanostructural materials obtained by intensive plastic deformation. Logos, Moscow, 2000, 272 pp. (In Russian).

16. Zhangjian Zhou, Yao Ma, Juan Du, Jochen Linke. Fabrication and characterization of ultra-fine grained tungsten by resistance sintering under ultra-high pressure. Materials Science and Engineering, A 505 (2009) 131-135.

17. E.D. Palik (Ed.), Handbook of Optical Constants of Solids, Academic Press, San Diego, California, 1985,1991 
18. V.Kh. Alimov, B. Tyburska-Püschel, S. Lindig, Y. Hatano, M. Balden, J. Roth, K. Isobe, M. Matsuyama, T. Yamanishi. Temperature dependence of surface morphology and deuterium retention in polycrystalline ITER-grade tungsten exposed to low-energy, high-flux D plasma. J. Nucl. Mater. 420 (2012) 519-524.

19. S. Lindig, M Balden, V Kh Alimov, T Yamanishi, WM Shu, J Roth. Subsurface morphology changes due to deuterium bombardment of tungsten. Phys. Scr. T138 (2009) 014040 (5 pp).

20. V.S. Voitsenya, M. Balden, A.I. Belyaeva, V.Kh. Alimov, B.Tyburska-Püschel, A.A. Galuza, A.A. Kasilov, I.V. Kolenov, V.G. Konovalov, O.O. Skoryk, S.I. Solodovchenko. Effect of sputtering on self-damaged recrystallized W mirror specimens. J. Nucl. Mater. 434 (2013) 375-381.

21. I.N. Evdokimov, V.E.Yurasova. Poverkhnost' №9 (1988) 5-22 (In Russian).

22. An American national standard ASME B46.1-1995, Surface texture (surface roughness, waviness, and lay), Jun. 14, 1996, The American Society of Mechanical Engineers, NY (USA), p.10.

23. G. Vayakis, E.R. Hodgson, V. Voitsenya, C.I. Walker. Fig. 18 in "Generic diagnostic issues for a burning plasma experiment”. Fusion Sci. Technol. 53 (2008) 699-750. 
Table 1. Calculated size of crystallites in the crystallized amorphous specimen found by processing the $\mathrm{x}$-rays diffractogram from [11].

\begin{tabular}{|c|c|c|}
\hline$\theta,^{\circ}$ & $\beta,^{\circ}$ & Size of crystallites, $\mathrm{nm}$ \\
\hline 13.5 & 0.125 & 65.2 \\
\hline 17.625 & 0.3 & 27.7 \\
\hline 18.95 & 0.17 & 49.3 \\
\hline 19.15 & 0.125 & 67.1 \\
\hline 20.05 & 0.17 & 49.6 \\
\hline 20.55 & 0.25 & 33.9 \\
\hline 21.85 & 0.17 & 50.2 \\
\hline 22.4 & 0.15 & 57.1 \\
\hline 23.75 & 0.15 & 57.7 \\
\hline 27.95 & 0.125 & 71.8 \\
\hline 30.45 & 0.15 & 61.3 \\
\hline 31.5 & 0.3 & 31.0 \\
\hline 32.05 & 0.17 & 55.0 \\
\hline
\end{tabular}

Table 2. Comparison of grain size and characteristic length of longitudinal inhomogeneity for all specimens studied. The scale of the longitudinal wavelength for the crystallized amorphous alloy is an estimate. For other types of mirrors the parameter $\mathrm{S}_{\mathrm{m}}$ is given.

\begin{tabular}{|c|c|c|}
\hline Specimen & $\begin{array}{c}\text { Size of } \\
\text { crystallites, } \mu \mathrm{m}\end{array}$ & $\begin{array}{c}\text { Scale of longitudinal } \\
\text { wavelength, } \mu \mathrm{m}\end{array}$ \\
\hline $\begin{array}{c}\text { Crystallized } \\
\text { amorphous alloy }\end{array}$ & $0.03-0.07$ & $\sim 1.0$ \\
\hline Rh film & $\sim 0.1$ & $0.15-0.3$ \\
\hline $\mathrm{Cu}$ alloy & $0.2-0.3$ & 6 \\
\hline Mo & $0.25-0.35$ & 4.8 \\
\hline $\mathrm{W}$ & $0.25-0.35$ & 3 \\
\hline ITER grade W & $1-3$ & 5.3 \\
\hline recrystallized W & $\geq 10$ & 150 \\
\hline
\end{tabular}




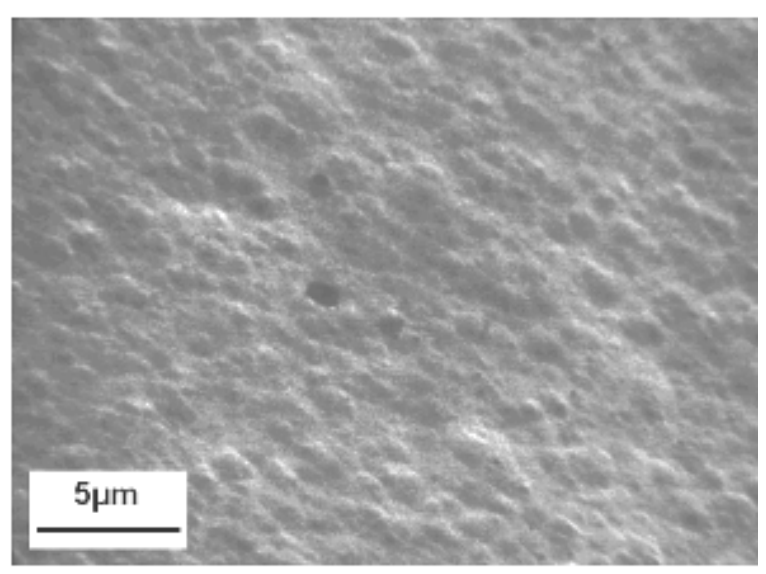

Fig.1. SEM image of the crystallized amorphous specimen after sputtering an $8.9 \mu \mathrm{m}$ thick layer by ions from an Ar plasma.

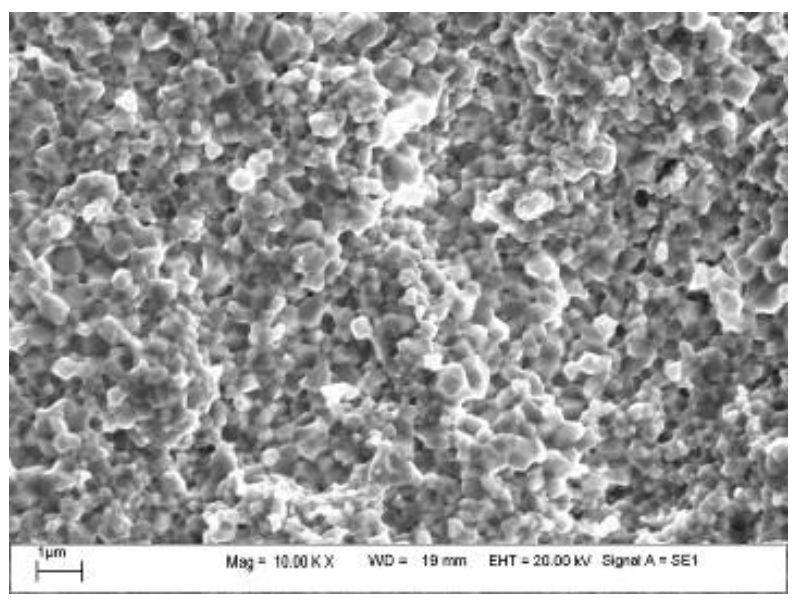

Fig.2. SEM image of fine-grain tungsten specimen fabricated by fast resistance sintering, but without surface preparation. 


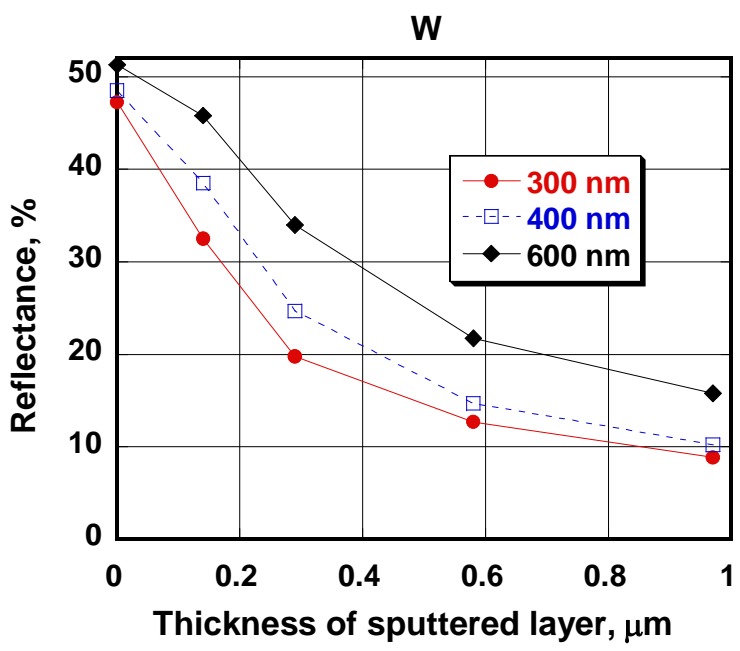

a)

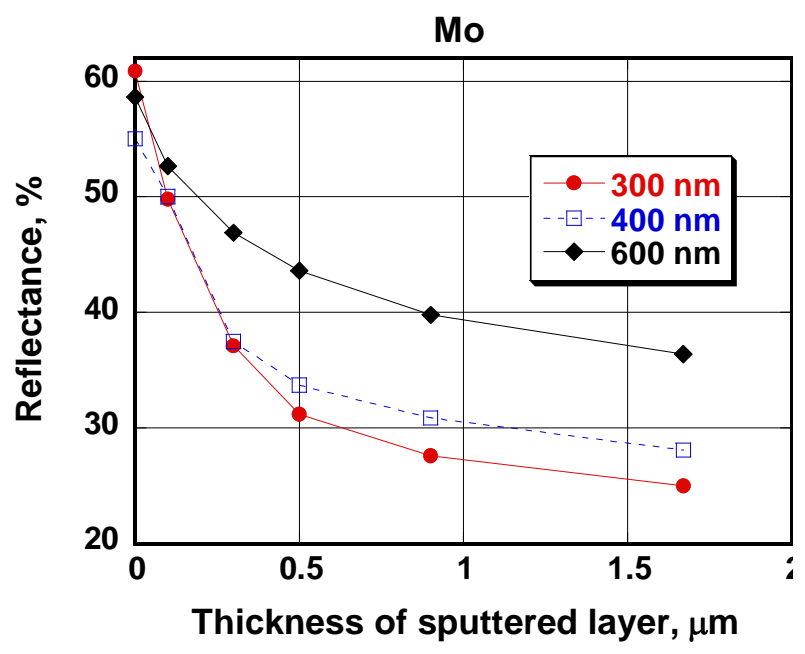

b)

Fig.3. Degradation of reflectance at the indicated wavelengths for fine-grain W (a) and Mo (b) mirror specimens as a function of the thickness of the sputtered layer (obtained by mass loss). Sputtering was performed with ions from argon (a) and deuterium (b) plasmas, respectively. In graph (b) the zero point of the y axis is suppressed. 


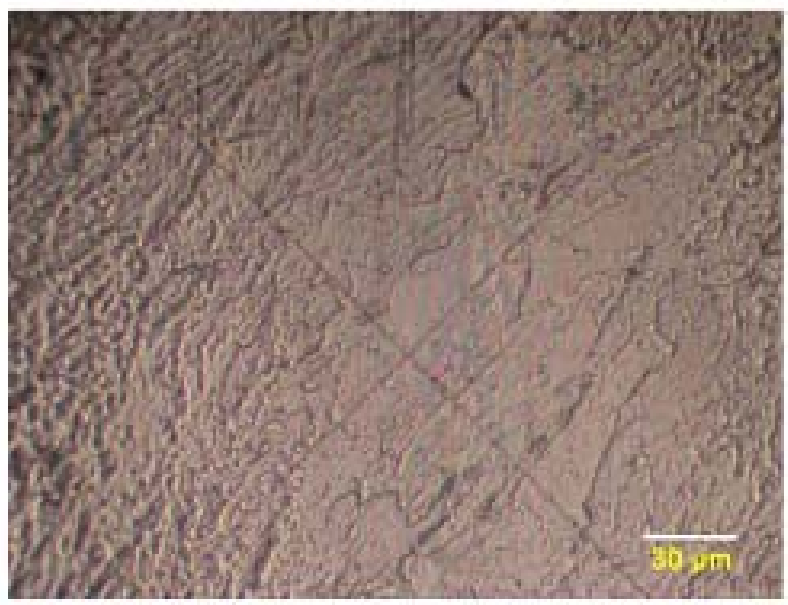

a)

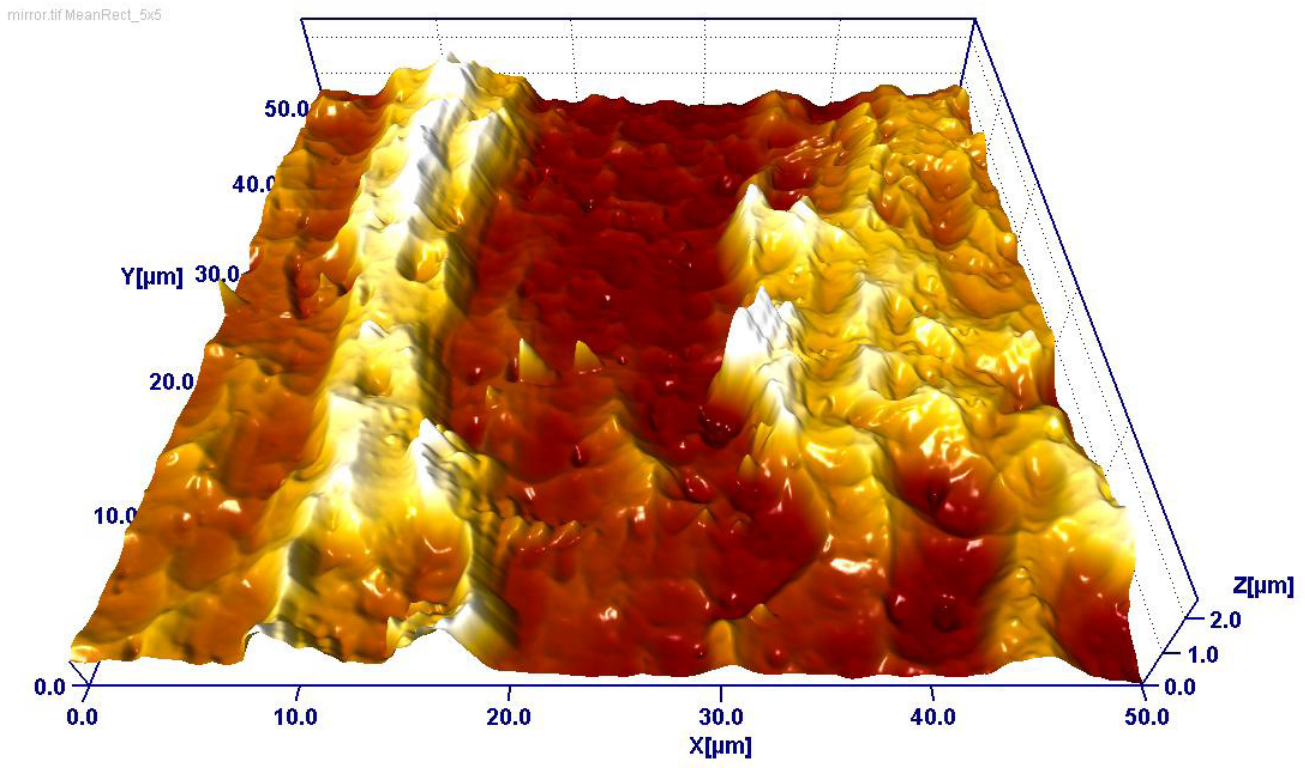

b)

Fig.4. The "ITER-grade" reference tungsten specimen after sputtering $\sim 4 \mu \mathrm{m}$ on average (according to the mass loss measurements) with ions from an argon plasma. a-optical micrograph, $b$ - AFM surface profile. 

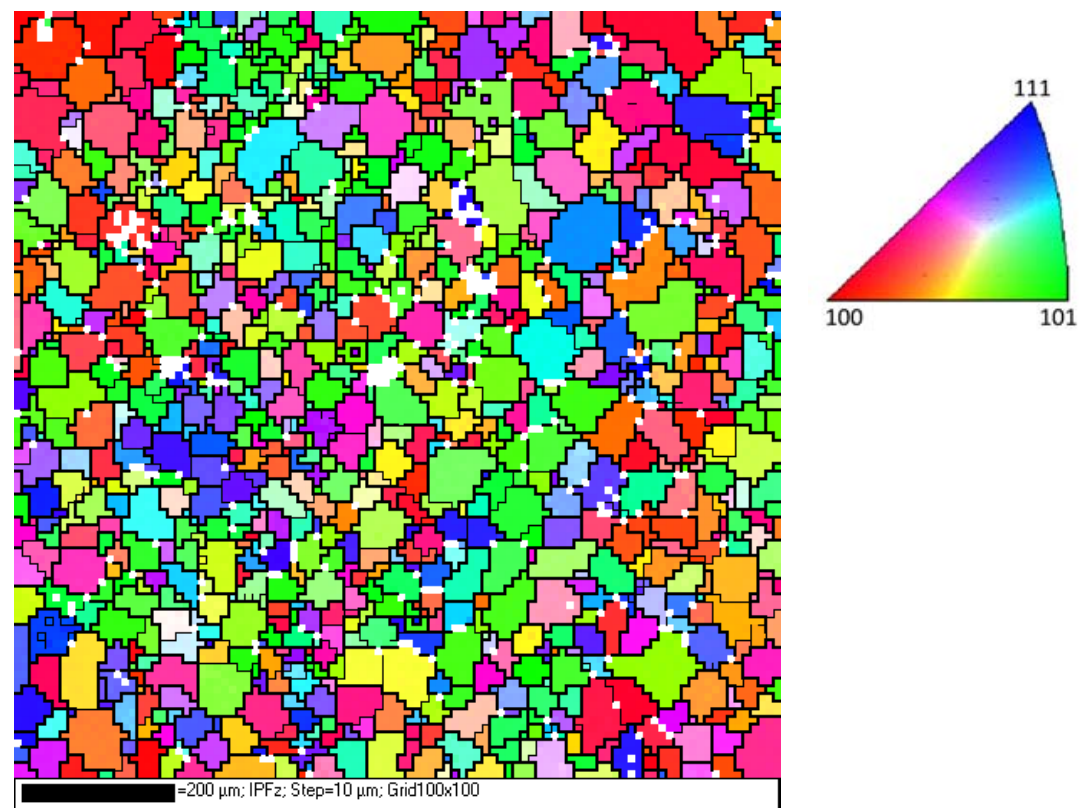

Fig.5. EBSD data for a $1 \times 1 \mathrm{~mm}^{2}$ region of the recrystallized $\mathrm{W}$ specimen indicating the orientations of grains perpendicular to the surface in the given color code.

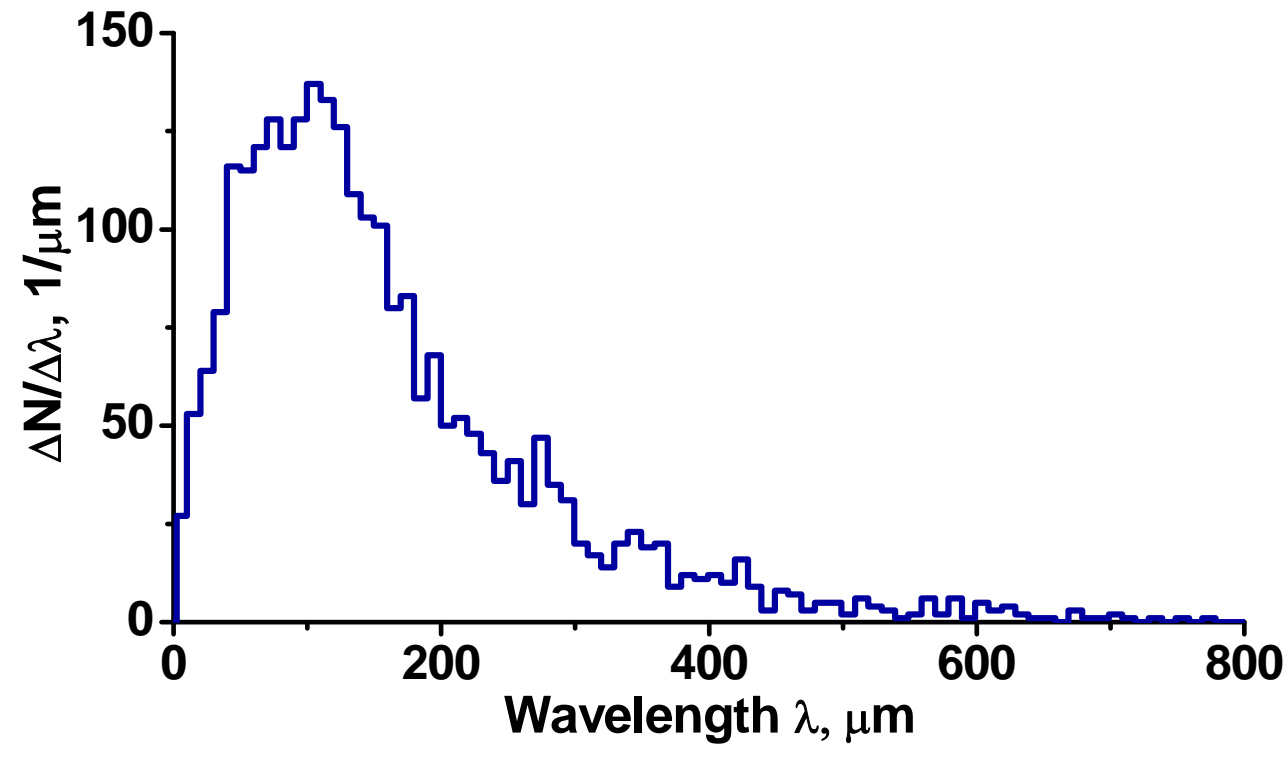

Fig. 6. The distribution of longitudinal periods for recrystallized W specimen. 


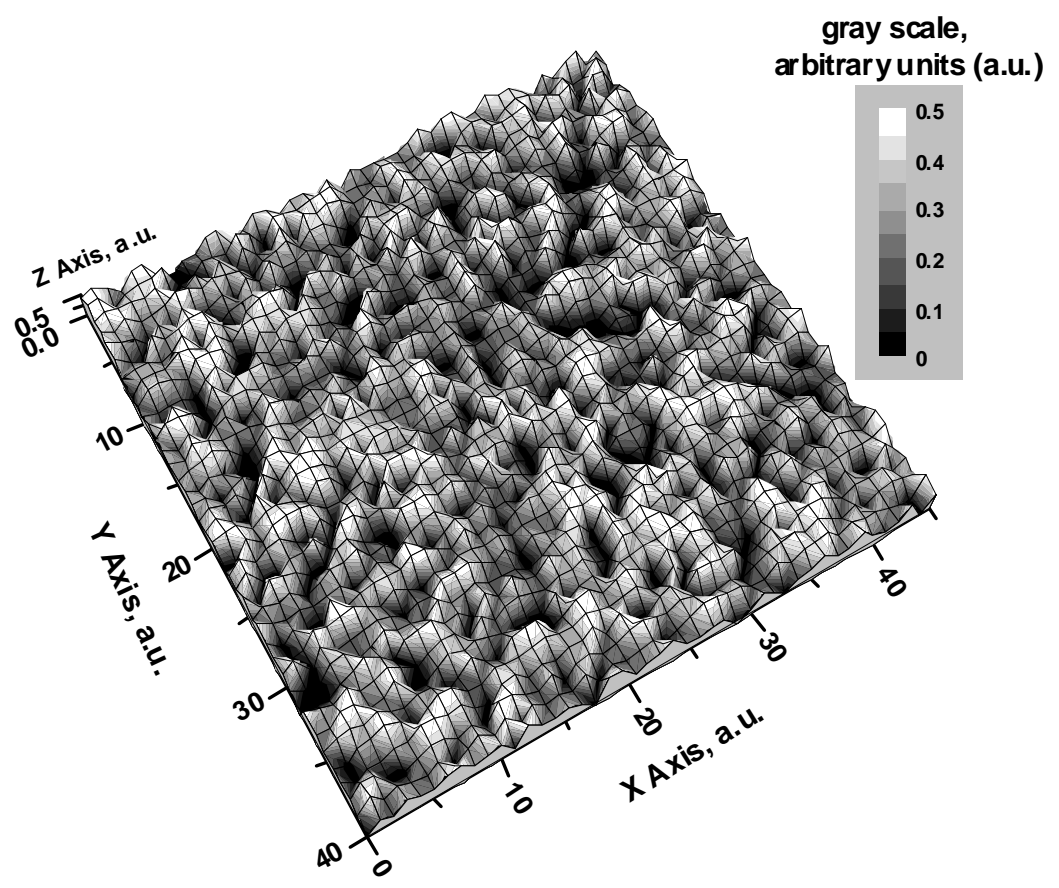

Fig.7. Modeled relief profile obtained for a $45 \times 40$ matrix of identically-sized crystallites with random orientations. Long-wave components are seen clearly.

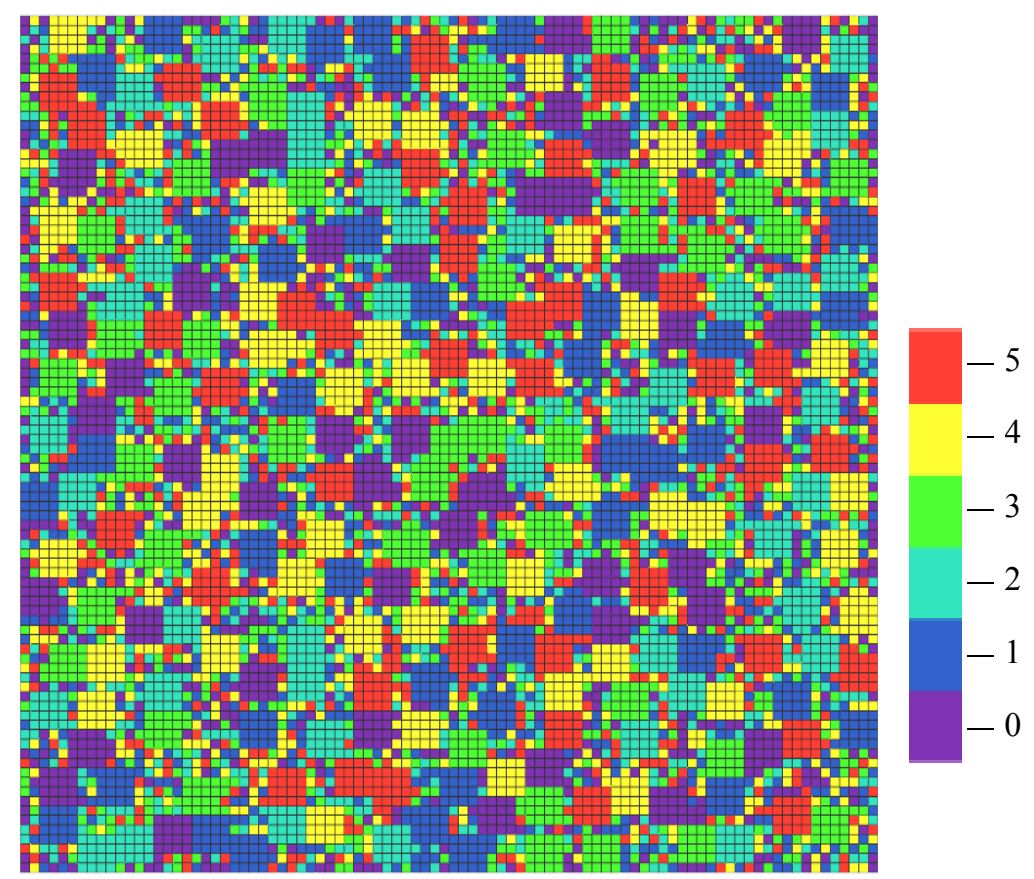

Fig.8. A color map of a "mirror" with randomly seeded cells having dimensions $4 \times 4$ and $1 \times 1$. Each color corresponds to the height of every cell on "mirror", in nm: 0 - violet, 1 - blue, 2 - cyan, 3 - green, 4 - yellow, 5 - red. 


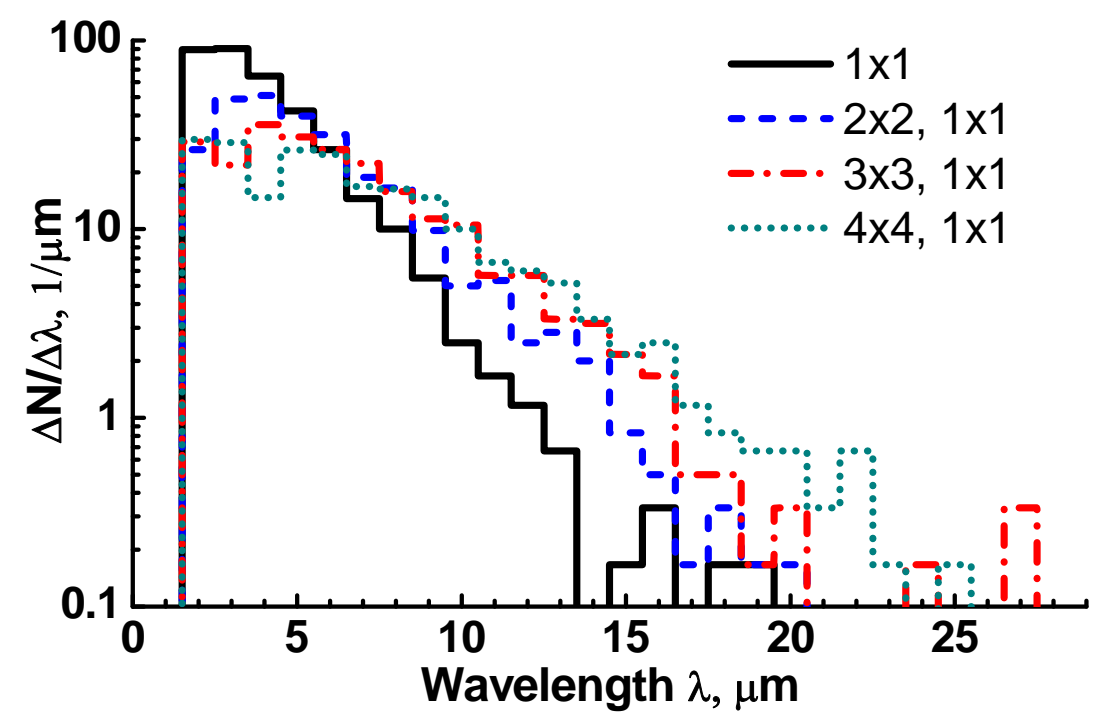

Fig. 9. The distributions with logarithm scale of longitudinal periods for cases i) $1 \times 1$, ii) $2 \times 2$, $1 \times 1$, iii) $3 \times 3,1 \times 1$, and iv) $4 \times 4,1 \times 1$.

\section{Appendix}

The analysis of surface roughness generally followed the procedure described in [8], which was created for the analysis of AFM data.

To provide meaningful values of $R_{q}$, the mean surface roughness, it is necessary to remove the influence of underlying large-scale variations in surface height. The trend-line approach is used for this purpose, with $\operatorname{tr}(x)=a x+b$ (using least squares) giving the trend line to the surface profile of heights, $h(x)$. If the slope $a=0$, the trend line simply gives the mean height. Higher polynomials, $\operatorname{tr}(x)=\Sigma a_{n} x^{n}$, with $n=2$ or 3 were also tested, but were not used due to the negligible values of $a_{2}$ and $a_{3}$.

$S_{m}$ is defined in [8], where the height profile is computed in a chosen direction on surface, along one straight line ("track") or along 10 parallel lines to have better statistics. The period $S_{i}$ is defined as the distance along a track between two crossings of the trend line, from below to above. This method is found to provide a much more reproducible measure of the surface roughness than that of "order-of-magnitude" estimates; e.g., from visual inspection of AFM images.

For our analysis, this method of calculating $S_{m}[8,22]$ was extended using Mathcad 13.0 to find the height profile $h(x)$ from a rectangular spiral path spreading from the center of the micrograph to the edges. This procedure gives much longer analysis paths providing better statistics. The program finds the beginning and end of each "oscillation" to calculate its length, $S_{i}$. The spiral path method was compared to the average over multiple parallel paths (e.g., a pixel row to the right alternating with the row to the left, etc), which gave similar results. 
Experimental measurements, e.g., AFM, include a noise component, composed of short wavelength "oscillations": sharp peaks, or larger-scale "goose skin" patterns. Inclusion of this noise in the analysis leads to an underestimate of the crystallites size. Typically, the noise r.m.s. height $R_{q, n o i s e}$ and its mean longitudinal period $S_{m, n o i s e}$ are much less than the crystallite sizes, and are excluded from our calculations by smoothing the profile $h(x)$. At a given location on the profile, values are smoothed by taking the median of the $m$ points centered on that value, with $m$ $=3-9$, with the condition that this changes the shape of the larger surface variations negligibly.

The distribution $\Delta N / \Delta \lambda$ is found by dividing the wavelength range into $M$ equal intervals of width $\Delta \lambda$ (i.e. "bins"). Each bin is centered at the wavelength $\lambda_{\mathrm{k}}$, which is the midpoint of the interval $\lambda_{\mathrm{k}}-\Delta \lambda / 2$ to $\lambda_{\mathrm{k}}+\Delta \lambda / 2$. $\Delta \mathrm{N}$ is the number of times an "oscillation" with length $\lambda_{\mathrm{k}}-\Delta \lambda / 2$ to $\lambda_{\mathrm{k}}+\Delta \lambda / 2$ occurs along a given profile. The value of $M$ is taken to be approximately $1 / 10$ of the number of points in a given profile.

An example of the calculated distribution $\Delta N / \Delta \lambda$ (number of longitudinal periods $\Delta N$ per wavelength interval $\Delta \lambda$ ) for one of the fine grain W mirror specimens with grain sizes in the range $250-350 \mathrm{~nm}\left(\mathrm{AFM}\right.$ area $\left.50 \times 50 \mu \mathrm{m}^{2}\right)$ without and with smoothing $(m=3)$ is shown in Fig.A1.

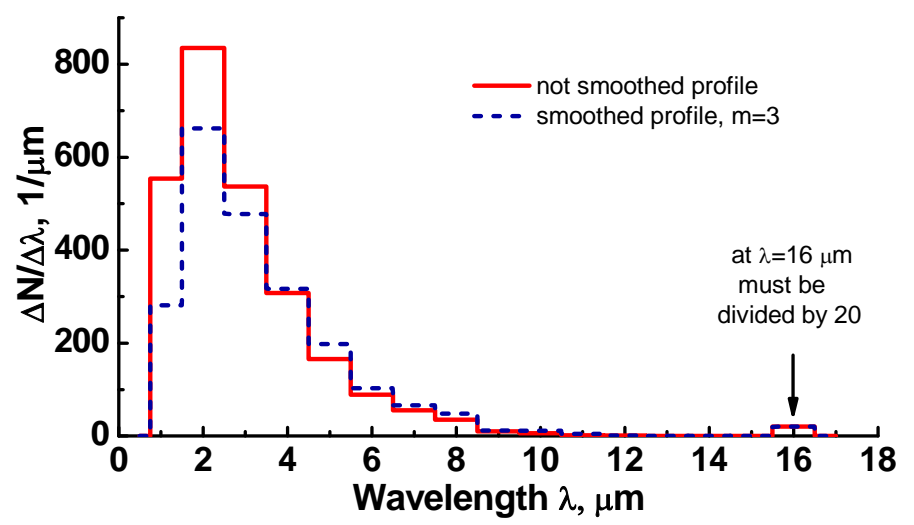

Fig. A1. The distribution of longitudinal periods with not smoothed profile $h(x)$ and after median smoothing. Note that the $\Delta N / \Delta \lambda$ value at $\lambda=16 \mu \mathrm{m}$ was multiplied by 20 to make it visible.

With $\mathrm{m}=3$ the magnitude of $\Delta \mathrm{N} / \Delta \lambda$ was reduced in interval $1 \leq \lambda \leq 3 \mu \mathrm{m}$, and at $\lambda \geq 4 \mu \mathrm{m}$ it increased slightly. In this example, the range of longitudinal wavelengths was $\lambda_{\mathrm{I}}=1-16 \mu \mathrm{m}$, with $S_{m}=2.7 \mu \mathrm{m}$ and the most probable wavelength $\lambda_{0}=2 \mu \mathrm{m}$ at the maximum of the distribution The roughness parameter $R_{q}=100 \mathrm{~nm}$ was found for this case.

Similar distributions with parameters $S_{m}, \lambda_{\mathrm{I}}$, and $\lambda_{0}$ were calculated for all AFM and STM microphotographs of mirrors presented in this article, excluding the crystallized amorphous mirrors. 
